\title{
Can Cognitive Therapy Be Conducted by Computers?
}

\author{
Tracy D. Eells ${ }^{1}$ • Marna S. Barrett ${ }^{2}$ - Jesse H. Wright ${ }^{1} \cdot$ Michael E. Thase $^{2}$
}

Published online: 24 September 2015

(C) Springer International Publishing AG 2015

\begin{abstract}
With consensus reached that psychotherapy is effective in treating many psychological disorders, researchers are developing more efficient and cost-effective ways to deliver evidence-based treatments. One avenue of exploration is computer-assisted therapy, which, if as effective as therapy delivered entirely by a therapist, could significantly reduce cost while expanding access to treatment, leveraging clinician time more advantageously, and increasing adherence to evidence-based techniques. This report examines research comparing computer-assisted therapy for major depression and anxiety disorders with controls and other treatments. Overall, results indicate moderate treatment effect sizes as compared to wait-list controls and equivalent results when compared to therapist-delivered treatment. However, dropout rates tend to be high with little clinician involvement and some studies show treatment effects attenuating in the long term. Additional and methodologically stronger studies are needed. Future directions may include integration of computer-
\end{abstract}

This article is part of the Topical Collection on Mood and Anxiety Disorders

Tracy D. Eells

eells@louisville.edu

Marna S. Barrett

msb@mail.med.upenn.edu

Jesse H. Wright

jwright@iglou.com

Michael E. Thase

thase@mail.med.upenn.edu

1 Department of Psychiatry and Behavioral Sciences, University of Louisville, 401 E. Chestnut Street, Suite 610, Louisville, KY 40202, USA

2 Mood \& Anxiety Disorders Treatment \& Research Program, Department of Psychiatry, University of Pennsylvania, 3535 Market Street, Suite 670, Philadelphia, PA 19104, USA assisted methods into clinics and training settings, capitalizing on opportunities that technology offers to improve treatment outcome and better educating clinicians about computerassisted treatments.

Keywords Major depression - Anxiety disorders · Psychotherapy · Psychological disorders · Computer-assisted therapy

\section{Introduction}

It is well established that psychotherapy is an effective treatment for a wide range of psychological disorders [1, 2]. Authoritative estimates are that $75 \%$ of patients undergoing therapy are better off after 4 months than those not receiving treatment [1]. Response rates for two of the most prevalent disorders [3-5], major depressive disorder and generalized anxiety disorder, typically range from 40 to $60 \%$, respectively, for those who participate in a minimally adequate course of psychotherapy. Cognitive behavior therapy (CBT) is prominent among these therapies and is the most extensively researched modality of therapy.

As psychotherapy research advances, researchers are exploring methods to disseminate effective evidence-based treatments in an efficient and cost-effective way. One effort has been the development of computer-assisted therapies. Computer-assisted therapy can be defined as "psychotherapy that utilizes a computer program to deliver a significant part of the therapy content or uses a computer program to assist the work of the therapist" [6]. The use of electronic technology may vary from minimal, in which it is considered an adjunct to therapy, to extensive, in which the computer completely replaces the therapist. 
In this review, we survey studies involving computerassisted cognitive behavior therapy (CCBT) for treatment of depression and anxiety. We focus on these two disorders because they have been the subjects of most research on computer-assisted psychotherapy. We focus on CBT since most computer-assisted models of therapy are based on CBT and because it has been shown to be an effective treatment approach. The goal of this review is to illustrate the range of interventions and how outcomes using these technologies compare with traditionally delivered treatments and untreated samples. Before surveying results, we provide a short history and discuss advantages and disadvantages of computerassisted therapy. We conclude with a discussion of future directions.

\section{History of Computers in Therapy}

Nearly 50 years ago, Joseph Weizenbaum [7] introduced ELIZA, perhaps the most famous computer program in clinical psychology. ELIZA was designed to simulate psychotherapy in a manner where people talk with the program as they would to a real therapist. ELIZA used therapeutic techniques similar to those of Carl Rogers [8], reflecting back to the person what the "patient" had just said. Despite the simplicity of the model, some people reported benefit from interacting with ELIZA and viewed the interaction as personal [9]. However, the program often misunderstood the user and gave feedback or instructions that were confusing or off target.

Since the development of ELIZA, others attempted to simulate therapeutic dialogue [e.g., 10] but concluded that computers are unlikely, at least in the foreseeable future, to replicate the interaction between a therapist and patient [11]. Attention therefore turned to the numerous ways in which computers and technology could benefit treatment. E-mail, video conferencing, texting, the Internet, and computer programs have been explored as ways to support treatment or as sole mechanisms to provide therapy [12].

Contemporary computer-assisted programs that address depression include Good Days Ahead (GDA) [13], Beating the Blues (BTB) [14], and Mood GYM [15]. Each is webbased, presents $\mathrm{CBT}$ in sequential modules, and includes interactive elements such as homework and other exercises and videos of individuals or characters modeling symptoms of major depression, as well as CBT strategies to manage depression. Although BTB and MoodGYM were developed to be delivered alone, that is, without a therapist, it is not uncommon for mental health professionals to oversee delivery of therapy. In contrast, GDA is designed to serve as an adjunct to clinician-delivered or clinician-supported treatment, such that the computer program and therapist form a "human-computer treatment team." (see Eells, Barrett, Wright, and Thase [16] for a more extensive summary of these programs).
Computer-assisted programs for the treatment of anxiety may vary more than those for depression. Programs may be characterized as predominantly self-help, minimal contact treatment, and predominantly therapist-delivered treatments in which computer technology augments treatment [17]. FearFighter, a nine-step Internet-based computer program incorporating therapist support for the treatment of agoraphobia and panic disorder [18], has been shown to be effective and equally so in comparison to standard exposure-based CBT [18].

\section{Advantages and Disadvantages of Computer-Assisted Psychotherapy}

Computer technology offers viable solutions to real problems. Psychotherapy is costly and requires significant amounts of therapist time. Manuals for delivering CBT for major depressive disorder typically call for 16 to 20 sessions over a period of 12 to 16 weeks [19]. If therapist time could be cut in half or even more by replacing therapy hours with a computer-based intervention and have a comparable outcome, the savings would be significant. Even more beneficial would be the increased efficiency of therapist time since two or more patients could be treated in the same amount of time as is currently the case for a single person. Computer-assisted therapies could also increase access to treatment. Internet and web-based programs are available to anyone at any time as long as there is an Internet connection. Such accessibility eliminates concerns about geographical barriers, transportation costs, travel time, and difficulties related to scheduling appointments.

Also, computer technology is well suited for delivering the psychoeducational components of treatment. Computer programs can be developed that deliver the core techniques of therapy in a standardized and consistent way from one patient to another. Sophisticated programs can deliver the rationale for treatment, empirically supported skill-building exercises, and homework suggestions all in an interactive and engaging way. Moreover, information shared by patients can be recorded and collected in such a way as to facilitate tracking of progress and providing additional opportunities for learning (e.g., repetition of core concepts, quizzes). Smart phones and similar devices can help extend treatment into life outside the therapy session via periodic, programmed reminders about skills such as deep breathing or a review of self-affirming coping statements.

Despite the many advantages to computer-assisted therapy, it is not without limitations and barriers. For instance, current computer-assisted psychotherapy programs do not contain the human connection that comes with therapist-delivered treatment. Many studies have shown that a genuine therapeutic relationship is an important component in predicting positive outcome [20]. Second, programs need to be designed that 
engage users, encourage adherence, and lead to an effective outcome that matches that of standard therapy. Third, moving from research-based program prototypes to widely and clinically available software is costly and depends upon the support and adoption of these technologies by clinicians. Clinicians may question the validity of these programs, view their use as a threat to clinical practice, and fear a lack of third-party payments for time spent monitoring patient use of the program or responding to patient queries. Finally, ethical and legal concerns arise when providing treatment for anxiety and depression via the Internet [21]. These include responding to potential suicidality and other types of dangerousness, maintaining confidentiality, and ensuring therapist competence in using emerging technologies. Legal issues may also arise with regard to licensure, which is state-based, and providing services to individuals who reside out of state. Thus, there are not only reasons to consider expanding the development of computer-assisted therapy, but also reasons to proceed with caution.

\section{Computer-Assisted Therapy for Depression}

A large number of studies of CCBT have been performed, and meta-analyses have confirmed the efficacy of computerassisted therapy for depressed outpatients [22-27]. Across meta-analyses of a progressively growing literature, CCBT has been shown to deliver moderate effect sizes for CCBT when compared to control groups. For example, Richards and Richardson [23] identified 19 randomized clinical trials published since 2001 that compared a computer-based psychological treatment for depression with a control condition, most of which were a wait-list or treatment as usual (TAU). All studies were published in peer-reviewed journals between March 2001 and March 2011 and included reliable and valid outcome measures assessing depression. Across all these studies, 1553 individuals were in treatment groups and 1443 were in control groups. Based on intent-to-treat analyses "where possible" (p. 330) in order to provide a conservative estimate of effect size, these authors found a moderate posttreatment pooled effect size of $d=0.56$ for self-reported depression. Across 14 studies that reported scores at follow-up, the average effect size comparing the computer-assisted intervention with the control condition was a more modest $d=0.20$. Further, as compared to control conditions, therapist-supported computerized interventions yielded better outcomes than those with no therapist support $(d=0.78)$, as did studies that provided only administrative support $(d=0.36)$. On the basis of a broader range of studies that were not restricted to randomized clinical trials (RCTs), the authors also found that computer-assisted interventions had high dropout rates, for programs involving no support (74\%) as compared to administrative support (38\%) and therapist support (28\%).
A meta-analysis by So and colleagues [22] explicitly focused on two issues of concern raised in the Richards and Richardson study. These are low effectiveness on long-term follow-up and high dropout rates. These researchers identified 14 randomized clinical trials (16 comparisons) comparing CCBT to either a wait-list or TAU. The studies met similar inclusion criteria as the Richards and Richardson study. They had to include a primary endpoint measure of depression at the outcome assessment immediately after intervention and a follow-up assessment of at least 6 months ( 5 of the 16 comparisons). A total of 2807 participants were included in the intent-to-treat analyses. Like Richards and Richardson, these researchers found an overall moderate effect size of 0.48 . They found an effect size of 0.63 for those studies employing wait-list controls and 0.23 for those using TAU as the control. In the five studies that conducted a long-term follow-up assessment, effect sizes dropped to 0.05 , showing no difference between participants receiving CCBT and those in the control conditions. Seven of the 16 comparisons met criteria for acceptable dropout rate $(<20 \%)$. The effect size for these studies was 0.59 . It is noteworthy that So et al. systematically excluded a study in which therapist involvement was more extensive in CCBT [i.e., 13]. This exclusion may have attenuated differences between treatment and comparison groups.

As seen above, most RCTs of CCBT for depression use control groups. Relatively few compare CCBT with standard CBT. Two notable exceptions are Selmi, Klein, Greist, Sorrell, and Erdman [28] and a series of studies by Wright and colleagues $[13,29,30]$. Selmi and colleagues conducted a RCT comparing a six-session interactive CBT program to therapistdelivered CBT and to wait-list controls. The patients were reliably diagnosed as depressed. After treatment and at 2month follow-up, both treatment groups had improved significantly more than the controls based on multiple symptom measures including the Beck Depression Inventory and the Hamilton Rating Scale for Depression.

Wright and colleagues [13] conducted a similar study using the GDA program, described earlier. Forty-five adults who were medication free and met criteria for DSM-IV nonpsychotic major depressive disorder were randomly assigned to cognitive therapy, computer-assisted therapy, or a wait-list control. Both active treatments consisted of nine sessions over 8 weeks. For the computer-assisted therapy, therapist time was reduced by one half after the first visit. Assessments were completed by independent raters before treatment, after 4 and 8 weeks of therapy, and 3 and 6 months posttreatment. All therapists were trained in cognitive therapy and followed the guidelines of Beck [31] for both treatment conditions. Patients in the CCBT condition saw the clinician for a brief session of $25 \mathrm{~min}$ and then worked alone on GDA. Results showed that both CCBT patients and those treated with standard CBT improved more than the wait-list controls, as assessed by both the 17-item Hamilton Depression Scale and 
the Beck Depression Inventory. Effect sizes at the end of treatment as measured by the Hamilton Rating Scale in intent-totreat analyses were 1.14 for the computer-assisted therapy and 1.04 for standard cognitive therapy. Evaluation of depression severity at 3 and 6 months posttreatment indicated that the patients maintained their treatment gains. A more recent, much larger, two-site study that included more severely and chronically depressed patients found similar acute phase results [29]. Follow-up results are yet to be reported.

Taken together, these studies show promising results for CCBT for depression, although not without qualifications. Further work is needed to better understand the role of moderators such as therapist assistance, specific characteristics of computerized programs, and symptom severity and chronicity in order to reduce dropout and improve both short- and longterm outcomes.

\section{Computer-Assisted Therapy for Anxiety}

Although less extensive than that for depression, more than 50 studies of computer-based treatments for anxiety disorders have been conducted including nine meta-analyses and 20 randomized controlled trials. These studies confirm the effectiveness of computer-assisted CBT in reducing patient anxiety in general and suggest its effectiveness with specific anxiety disorders. A recent meta-analysis by Grist and Cavanagh [32] examined 49 randomized controlled trials comparing computer-assisted CBT to other therapies $(n=24)$ and to wait-list controls $(n=25)$. Computer-assisted CBT was found to be significantly more effective than both wait-list and active control conditions (overall mean effect size of $g=0.77$ ), but this finding was for a wide range of mental health disorders that included anxiety.

The Gale and Cavanagh study highlights one of several factors limiting conclusions about the effectiveness of computer-assisted CBT for anxiety disorders. That is, few studies focus on only one anxiety disorder rather than a combination of disorders. Moreover, studies vary in how the computer-assisted program is delivered (i.e., Internet, standalone PC, or palmtop computer). A 2009 meta-analytic review [33] attempted to address these limitations by pooling together all types of programs and delivery systems as well as different types of anxiety disorders. Across 13 randomized controlled trials (RCTs) comparing computer-assisted treatment with face-to-face treatment, essentially no difference was found between treatments $(d=-0.06)$ across all anxiety disorders (panic disorder/agoraphobia, social phobia, specific phobia, OCD, PTSD, generalized anxiety disorder (GAD)). Further analysis failed to identify differences between conditions for type of disorder, although the authors note that most of these studies did not include clinical samples but those recruited from the community.
An earlier meta-analysis by Spek and colleagues [25] examined 12 studies of Internet-based CBT. They found a relatively large mean effect size $(d=0.96)$ for anxiety disorders when compared with control groups. Additionally, the presence of therapist support in the computer-based treatments produced a larger mean effect size than when no therapist support was provided $(d=0.26)$. However, type of anxiety disorder was not examined independently nor did the study include comparisons of a computer-assisted program to traditional face-to-face therapy.

Reger and Gahm [34] conducted a meta-analysis of 19 studies in which Internet- or computer-based treatment was compared to therapist-delivered treatment. Like previous studies, the findings revealed equivalent effects across anxiety disorders ( $d \mathrm{~s}$ ranged from 0.49 to 1.14 ), although these conclusions were limited by small sample sizes, lack of placebo controls, and too few studies to examine specific disorder type.

In regard to randomized controlled trials, almost all of the studies that we reviewed compared Internet- or computerassisted treatments to a wait-list or no treatment control rather than to another treatment. In these studies, the computerassisted treatment was found to be effective for all anxiety disorders examined, including posttraumatic stress disorder [35], social phobia [36], panic [37-39], and GAD [40, 41]. Although relatively few in number, studies directly comparing computer-assisted treatment with another treatment demonstrated the efficacy of computer-assisted treatments. For example, Litz, Engel, Bryant, and Papa [42] compared an 8week Internet-based self-management CBT to Internet-based supportive counseling for PTSD. Self-management CBT was more effective in reducing PTSD symptoms and depression scores as well as improving overall functioning. Robinson, Titov, Andrews, McIntyre, Schwencke, and Solley (2010) [43] found equivalent outcomes when comparing clinicianassisted CBT to face-to-face CBT for the treatment of GAD.

As noted above, an early Internet-based program of exposure therapy, FearFighter, is a nine-step program for the treatment of agoraphobia and panic disorder [18]. In the first randomized controlled trial of Fear Fighter, 93 outpatients with phobia or panic disorder were randomized to one of three conditions: traditional exposure therapy with a clinician, self-exposure therapy with FearFighter, or a mainly computer-guided self-relaxation program. Brief coaching and review of progress were provided by a clinician in each of the two computer conditions. Although more patients dropped out of the two computer groups (FearFighter and relaxation), patients treated by FearFighter reported similar improvement to those patients treated by traditional clinician-guided exposure. Moreover, this equivalency was obtained despite $73 \%$ less clinician time for those treated by FearFighter. [18]

Viewing these studies in whole, evidence supports the conclusion that Internet-based and computer-assisted CBT is 
effective in reducing patient anxiety across a number of specific disorders. Further, it produces an effect similar to that of traditional face-to-face therapy.

\section{Future Directions}

Studies to date suggest that computer-assisted CBT may offer a treatment that is as effective and requires less therapist time and involvement than traditional CBT. Nevertheless, CCBT still is used infrequently in clinical practice and has not yet had much impact on the overall delivery of mental health services. Why would an effective treatment that shows distinctive cost-benefits [44] along with improved access not be widely implemented? Resistance of clinicians to a method that they might believe would reduce need for their services could be one reason. Also, the volume of research on CCBT is still relatively modest in comparison to the large number of studies on standard CBT. Perhaps, an expected general increase in research on CCBT will provide a more convincing (or less convincing) case for broader dissemination. However, the processes needed for effective wide-spread implementation have received scant attention.

Implementation and dissemination efforts are important next steps in CCBT research. A target for such studies could be the lack of well-developed systems in either mental health or primary care settings to support the delivery of CCBT. Research on delivery models that can be sustained after an investigation has been completed is clearly needed. And, detailing of specific implementation strategies could be part of this research. Examples of such strategies include the following: (1) weaving CCBT into the culture and clinical workload of mental health and primary care settings, (2) training clinicians to support CCBT (a method that is not taught currently), (3) developing clinician dashboards to track progress and guide treatment, (4) designing methods to inform and recruit patients to participate in CCBT, (5) providing access to disadvantaged persons who may not have Internet experience or availability, and (6) investigating reimbursement and licensing issues for computer-assisted treatment.

Another need in CCBT research is to gain a better understanding of the clinician's role in treatment delivery. As noted in our review, CCBT alone, without any support from a clinician, has had small effect sizes in comparison to CCBT with clinician involvement. Head-to-head studies of CCBT versus standard CBT have not shown an erosion of effectiveness when clinician contact is reduced substantially in computer-assisted CBT $[13,28]$. Yet, there has been a paucity of direct comparisons between computer-assisted and standard CBT - studies that are needed to explore possible differences in the therapeutic relationship in these different models for treatment delivery. One investigator found a negative effect on treatment alliance when therapy is delivered electronically [45], although others found similar results as with standard therapy, at least in early alliance ratings [46]. Notably, patients typically rate CCBT as a credible and acceptable treatment method $[18,30]$. A recently completed, large-scale trial of CCBT versus CBT collected data on the therapeutic alliance in both forms of treatment [29]. Awaited data analyses from this study will start to provide information on the impact, if any, of adding technology to the treatment relationship.

Other parameters of clinician activity and effectiveness in CCBT that need more research are the amount, method of delivery, and technique of treatment. In a review of CCBT conducted for a meta-analysis of CCBT for major depression, Thase and colleagues [47] found a wide variety of time spent by the clinician in supporting treatment (from less than an hour to $14 \mathrm{~h}$, with most studies between 1 and $4 \mathrm{~h}$ ). No clear relationship was found between clinician support time and outcome. But, the clinical populations were diverse (some mildly depressed recruited over the Internet without requirement for diagnosis of major depression and some with clinically recruited patients with SCID diagnosis of major depression), as were the computer programs used for treatment (some primarily text-based with little or no interactivity and some with sophisticated, multimedia interfaces and high levels of interactivity). Thus, important questions about clinician involvement in CCBT remain. How much clinician time is needed for effective treatment? Does the method of communication (e.g., face-to-face, telephone, e-mail, other) make a difference? Is it important for the clinician to be trained in CBT and deliver part of the therapy using standard CBT methods to achieve the best benefit, or can the support method primarily focus on coaching on use of the computer program? What clinician support methods are most useful in promoting adherence and positive outcome in CCBT?

Among other possible future directions for CCBT, technological advances are likely to fuel greater use of computerrelated methods in clinical practice. Although clinicians currently may use computers primarily for electronic medical records or for Internet searches, programs for CCBT will likely become increasingly interactive and appealing to both patients and clinicians. Some of the current opportunities are to construct programs that are more customized to meet the needs of individual patients, are "stickier" (more likely to have users want to stay with the program to completion), use mobile delivery, and provide better interfaces to integrate CCBT with other elements of treatment. Because of the rapid pace of technological development, the horizon for CCBT is impossible to predict. However, it is possible that the early work on ELIZA — an attempt to construct a meaningful therapeutic dialogue between human and computer-presaged a future form of psychotherapy. 


\section{Conclusion}

In sum, computer-assisted CBT may offer treatments for depression and anxiety that are as effective and require less time and less therapist involvement than traditional models of therapy. These models can also provide greater access to mental health care for those in rural or underserved areas as well as those with special needs (e.g., HIV+, pregnancy, agoraphobia, etc.). But, future research is warranted to address concerns about effectiveness for specific disorders, delivery methods, cost-effectiveness, clinician involvement, and acceptance.

\section{Compliance with Ethics Guidelines}

Conflict of Interest Jesse Wright has cited grants and stock as conflicts. Marna Barrett and Michael E Thase have no conflicts. Tracy Eells listed a grant from the National Institutes of Mental Health.

Jesse H. Wright, M.D., Ph.D., has an equity interest in Empower Interactive and Mindstreet LLC, developers of software used in his research on computer-assisted CBT. This conflict of interest is managed with a plan with the University of Louisville.

Human and Animal Rights and Informed Consent This article does not contain any studies with human or animal subjects performed by the author.

\section{References}

1. Lambert MJ. The efficacy and effectiveness of psychotherapy. In: Lambert MJ, editor. Bergin and Garfield's handbook of psychotherapy and behavior change. 6th ed. New York: Wiley; 2013. p. 169218.

2. Wampold BE. The great psychotherapy debate: the evidence for what makes psychotherapy work. New York: Routledge; 2015.

3. Kessler RC, Berglund P, Demler O, Jin R, Koretz D, Merikangas $\mathrm{KR}$, et al. The epidemiology of major depressive disorder: results from the National Comorbidity Survey Replication (NCS-R). J Am Med Assoc. 2003;289:3095-105.

4. Kessler RC, Wang PS. Epidemiology of depression. In Handbook of depression. 2nd ed. New York: Guilford Press; 2009. p. 5-22.

5. Kessler RC, Ruscio AM, Shear K, Wittchen H-U. Epidemiology of anxiety disorders. Curr Top Behav Neurosci. 2010;2:231-5.

6. Wright JH, Beck AT, Thase ME. Cognitive therapy. In: Hales R, Yudofsky S, Gabbard G, editors. American psychiatric publishing textbook of psychiatry. 5th ed. Arlington: American Psychiatric Publishing; 2008. p. 1211-56.

7. Weizenbaum J. ELIZA - a computer program for the study of natural language communication between man and machine. Commun Assoc Comput Mach. 1966;9:36-45.

8. Rogers CR. Client-centered therapy, its current practice, implications, and theory. Boston: Houghton Mifflin; 1951.

9. O’Dell JW, Dickson J. Eliza as a "therapeutic" tool. J Clin Psychol. 1984;40:942-5.

10. Colby KM, Colby PM. Dialogues in natural language with GURU, a psychologic inference engine. Philos Psychol. 1990;3.

11. Bloom BL. Computer-assisted psychological intervention: a review and commentary. Clin Psychol Rev. 1992;12:169-97.

12. Barnett J. Utilizing technological innovations to enhance psychotherapy supervision, training, and outcomes. Psychotherapy. 2011;48:103-8.
13. Wright JH, Wright AS, Albano AM, Basco MR, Goldsmith LJ, Raffield $\mathrm{T}$, et al. Computer-assisted cognitive therapy for depression: maintaining efficacy while reducing therapist time. Am J Psychiatry. 2005;162:1158-64.

14. Proudfoot J, Ryden C, Everitt B, Shapiro DA, Goldberg D, Mann A, et al. Clinical efficacy of computerised cognitive-behavioural therapy for anxiety and depression in primary care: randomised controlled trial. Br J Psychiatry. 2004;185:46-54.

15. Christensen H, Griffiths KM, Jorm AF. Delivering interventions for depression by using the internet: randomised controlled trial. $\mathrm{Br}$ Med J. 2004;328:5.

16. Eells TD, Barrett MS, Wright JH, Thase M. Computer-assisted cognitive-behavior therapy for depression. Psychotherapy. 2014;51:191-7.

17. Newman MG, Szkodny LE, Llera SJ, Przeworski A. A review of technology-assisted self-help and minimal contact therapies for anxiety and depression: is human contact necessary for therapeutic efficacy? Clin Psychol Rev. 2011;31:89-103.

18. Marks IM, Kenwright M, McDonough M, Whittaker M, MataixCols D. Saving clinicians' time by delegating routine aspects of therapy to a computer: a randomized controlled trial in phobia/ panic disorder. Psychol Med. 2004;34:9-17.

19. Craighead WE, Sheets ES, Brosse AL, Ilardi SS. Psychosocial treatments for major depressive disorder. In: Nathan PE, Gorman JM, editors. A guide to treatments that work. 3rd ed. New York: Oxford University Press; 2007. p. 289-307.

20. Norcross JC, editor. Psychotherapy relationships that work: evidence-based responsiveness, 2nd edition. New York: Oxford University Press; 2011.

21. Fisher CB, Fried AL. Internet-mediated psychological services and the American psychological association ethics code. Psychother Theory Res Pract Train. 2003;40:103-11.

22. So M, Yamaguchi S, Hashimoto S, Sado M, Furukawa TA, McCrone P. Is computerised CBT really helpful for adult depression? - a meta-analytic re-evaluation of CCBT for adult depression in terms of clinical implementation and methodological validity. BMC Psychiatry. 2013;13:1-14.

23. Richards D, Richardson T. Computer-based psychological treatments for depression: a systematic review and meta-analysis. Clin Psychol Rev. 2012;32:329-42.

24. Andersson G, Cuijpers P. Internet-based and other computerized psychological treatments for adult depression: a meta-analysis. Cogn Behav Ther. 2009;38:196-205.

25. Spek V, Cuijpers P, Nyklíček I, Riper H, Keyzer J, Pop V. Internetbased cognitive behaviour therapy for symptoms of depression and anxiety: a meta-analysis. Psychol Med. 2007;37:319-28.

26. Barak A, Hen L, Boniel-Nissim M, Shapira NA. A comprehensive review and a meta-analysis of the effectiveness of internet-based psychotherapeutic interventions. J Technol Hum Serv. 2008;26: 109-60.

27. Andrews G, Cuijpers P, Craske MG, McEvoy P, Titov N. Computer therapy for the anxiety and depressive disorders Is effective, acceptable and practical health care: a meta-analysis. PLoS ONE. 2010;5, e13196.

28. Selmi P, Klein M, Greist J, Sorrell S, Erdman H. Computeradministered cognitive-behavioral therapy for depression. Am J Psychiatry. 1990;147:51-6.

29. Wright JH, Brown G, Eells TD, Wisniewski S, Barrett MS, Balasubramani GK, et al. Computer-assisted cognitive-behavior therapy for depression: acute treatment phase outcome. Chicago: In 2014 National Network of Depression Centers Annual Conference; 2014.

30. Wright JH, Wright AS, Salmon P, Beck AT, Kuykendall J, Goldsmith LJ, et al. Development and initial testing of a multiprogram for computer-assisted cognitive therapy. Am J Psychother. 2002;56:76-84. 
31. Beck JS. Cognitive therapy: basics and beyond. New York: Guilford Press; 1995.

32. Grist R, Cavanagh K. Computerised cognitive behavioural therapy for common mental health disorders, what works, for whom under what circumstances? A systematic review and meta-analysis. J Contemp Psychother. 2013;43:243-51.

33. Cuijpers P, Marks I, van Straten AM, Cavanagh K, Gega L, Andersson G. Computer-aided psychotherapy for anxiety disorders: a meta-analytic review. Cogn Behav Ther. 2009;66-82.

34. Reger MA, Gahm GA. A meta-analysis of the effects of internetand computer-based cognitive-behavioral treatments for anxiety. $\mathrm{J}$ Clin Psychol. 2009;65:53-75.

35. Knaevelsrud C, Maercker A. Long-term effects of an internet-based treatment for posttraumatic stress. Cognitive Behavior Therapy. 2010;39:72-7.

36. Titov N, Andrews G, Schwencke G, Robinson E, Peters L, Spence J. Randomized controlled trial of Internet cognitive behavioural treatment for social phobia with and without motivational enhancement strategies. Aust N Z J Psychiatry. 2010;44:938-45.

37. Carlbring P, Westling BE, Ljungstrand P, Ekselius L, Andersson G. Treatment of panic disorder via the Internet: a randomized trial of a self-help program. Behav Ther. 2001;32:751-64.

38. McCrone P, Marks IM, Mataix-Cols D, Kenwright M, McDonough M. Computer-aided self-exposure therapy for phobia/panic disorder: a pilot economic evaluation. Cognitive Behavior Therapy. 2009;38:91-9.

39. Newman MG, Kenardy J, Herman S, Taylor CB. Comparison of palmtop-computer-assisted brief cognitive-behavioral treatment to cognitive-behavioral treatment for panic disorder. J Consult Clin Psychol. 1977;65:178-83.

40. Paxling B, Almlov J, Dahlin M, Carlbring P, Breitholtz E, Eriksson $\mathrm{T}$, et al. Guided internet-delivered cognitive behavior therapy for generalized anxiety disorder: a randomized controlled trial. Cogn Behav Ther. 2011;40.

41. Titov N, Andrews G, Robinson E, Schwencke G, Johnston L, Solley $\mathrm{K}$, et al. Clinician-assisted Internet-based treatment is effective for generalized anxiety disorder: a randomized controlled trial. Aust N Z J Psychiatry. 2009;43:905-12.

42. Litz B, Engel C, Bryant R, Papa A. A randomized, controlled proofof-concept trial of an internet-based, therapist-assisted self-management treatment for posttraumatic stress disorder. Am J Psychiatry. 2007;164:1676-83.

43. Robinson, E., Titov, N., Andrews, G., McIntyre, K., Schwencke, G., and Solley, K. Internet treatment for generalized anxiety disorder: a randomized controlled trial comparing clinician vs. technician assistance. PLoS ONE. 2010, 5.

44. McCrone, P., Knapp, M., Proudfoot, J., Ryden, C., Cavanagh, K., Shapiro, D.A., Ilson, S., Gray, J.A., Goldberg, D., Mann, A., et al. Cost-effectiveness of computerised cognitive-behavioural therapy for anxiety and depression in primary care: randomised controlled trial, 2004. 185 .

45. Sucala M, Schnur JB, Constantino MJ, Miller SJ, Brackman EH, Montgomery GH. The therapeutic relationship in e-therapy for mental health: a systematic review. J Med Int Res. 2012;14, e110.

46. Andersson G, Paxling B, Wiwe M, Vernmark K, Felix CB, Lundborg L, et al. Therapeutic alliance in guided internetdelivered cognitive behavioural treatment of depression, generalized anxiety disorder and social anxiety disorder. Behav Res Ther. 2012;50:544-50.

47. Thase ME, Wisniewski S, Brown G, Eells TD, Barrett MS, Wright $\mathrm{JH}$. Meta-analysis of computer-assisted cognitive-behavior therapy for depression: update and extension. Chicago: In 2014 National Network of Depression Centers Annual Conference; 2014. 